\title{
EFEITO DA ADUBAÇÃO FOSFATADA E DA CALAGEM NAS RELAÇÕES DE INTERFERÊNCIA ENTRE PLANTAS DE SOJA E CAPIM-MARMELADA ${ }^{1}$
}

\author{
DAGOBERTO MARTINS ${ }^{2}$ e ROBINSON A. PITELLI ${ }^{3}$
}

\section{RESUMO}

Com o objetivo de estudar os efeitos da calagem e da adubação fosfatada sobre $o$ crescimento de plantas de soja e capim-marmelada e, o reflexo destas práticas nas relações de interferência entre a planta daninha e a planta cultivada. $\mathrm{O}$ estudo foi conduzido em casa-devegetação por um período de 49 dias. Utilizaramse vasos de 4 litros, contendo substrato retirado de um LR-distrófico. O delineamento experimental adotado foi o inteiramente casualizado, com quatro repetições. Os tratamentos foram dispostos em um esquema fatorial $2 \times 3 \times 4$, onde tinha-se: dois níveis de calagem (presença e ausência), três condições de vegetação nos vasos (soja cultivada isolada, planta daninha cultivada isolada e a convivência das espécies) e quatro doses de aplicação de fósforo (0, 50,100 e 200 ppm) no substrato. A calagem incrementou a altura, o número de trifólios, os teores de clorofila $\mathbf{a}$ e $\mathbf{b}$, a biomassa seca e a área foliar das plantas de soja e, proporcionou decréscimos na altura, no número de perfilhos e no acúmulo de matéria seca de plantas de capim-marmelada. A interferência imposta pelo capim-marmelada reduziu a altura de plantas, o número de trifólios, os teores de clorofila a, o acúmulo de matéria seca e a área foliar das plantas de soja. De forma oposta, a competição imposta pela soja determinou decréscimos na altura, no número de perfilhos, nos teores de clorofila $\mathbf{a}$ e b, no acúmulo de matéria seca e na área foliar das plantas de capim-marmelada. Já, a adubação fosfatada incrementou a altura, o número de perfilhos, o acúmulo de matéria seca e a área foliar das plantas de capim-marmelada.

Palavras chave: Glycine max, Brachiaria plantaginea, competição, fósforo, calcário.

\section{ABSTRACT \\ Effects of liming and phosphorus fertilization on interference between soybean and alexander grass}

The present work studied the effects of soils liming and phosphorus fertilization on interespecific interference involving soybean and Alexander grass under greenhouse conditions. The experimental design was completely randomized with four replications. The treatments were amauged in factorial design $2 \times 2 \times 4$ for each species with two conditions (species groueing alone or interespecific competition), two liming condition (none $\mathrm{e}$ with) and four levels of phosphorus fertilizations added to substrate $(0,50$, 100 and $200 \mathrm{ppm}$ ). Interference of Alexander grass reduced height, leaf number, leaf area, total dry matter accumulation. Soy beam growth was increased by liming and phosphorus fertilization; however, Alexander grass growth was increased only by phosphorus fertilization. Liming and phosphorus fertilization increased height, leaf number, leaf area and dry matter accumulation in soy beam. Phosphorus fertilization increased height, tiller number and total dry matter in Alexander grass. As the phosphorus fertilization increased the interference of Alexander grass also increased.

Key words: Glycine max, Brachiaria plantaginea, competition, lime.

\footnotetext{
${ }^{1}$ Recebido para publicação em 18/06/99 e na forma revisada em 30/10/2000.

${ }^{2}$ Prof $^{\circ}$ Dr., Dept ${ }^{\circ}$ de Produção Vegetal, FCA/UNESP, C.P. 237, CEP: 18603-970, Botucatu/SP.

${ }^{3}$ Prof $^{\mathrm{o}}$ Titular, Dept ${ }^{\mathrm{o}}$ de Biologia Aplicada à Agropecuária, FCACVJ/UNESP, CEP: 14870-000, Jaboticabal/SP.
} 


\section{INTRODUÇÃO}

Com a implantação dos agroecossistemas, o equilíbrio obtido durante o processo evolutivo pelas populações que se sucediam foi comprido e, especialmente com as monoculturas que não conseguem cobrir totalmente os nichos existentes, dando oportunidade para o surgimento de plantas com características pioneiras, as plantas daninhas que são extremamente eficientes no estabelecimento e reprodução nesses ambientes (Pitelli, 1985).

Com o desenvolvimento da agricultura, as plantas daninhas também evoluíram, tanto nos mecanismos adaptativos das populações existentes como no aparecimento de novas espécies e, especialmente nas áreas de fronteira agrícola.

Devido as características peculiares de cada cultivo agrícola, como região geográfica, sistema de plantio, cobertura de solo e época de semeadura, ocorreu uma certa especialização nas comunidades infestantes que convivem com as culturas conduzidas pelo homem nas diferentes regiões do globo. Tal especialização tornou-se muito mais evidente com o aparecimento dos herbicidas seletivos. Como exemplo tem-se o capim-marmelada [Brachiaria plantaginea (LINK) Hitch.] na cultura da soja, onde a região Sul do Brasil predomina nas comunidades infestantes (Kissmann, 1991 e Martins, 1994).

A predominância de uma espécie, como esta, deve-se à grande adaptação do clima regional, à época de semeadura e ao ciclo agrícola da soja, à características edáficas proporcionadas à planta cultivada e, em especial, à relativa tolerância desta espécie de planta daninha aos herbicidas utilizados na cultura.

Dentre as características edáficas proporcionadas à cultura da soja, destacam-se a correção do $\mathrm{pH}$ do solo e a complementação do nível de fósforo disponível no solo. Estas práticas provavelmente modificam o crescimento das plantas daninhas e, com isso, podem influenciar no balanço sobre as plantas de soja (Buckanan et al., 1975; Gilbert \& Pember, 1975 e Michelini, 1983).
Assim, a presente pesquisa procurou estudar os efeitos da calagem e da adubação fosfatada sobre o crescimento inicial de plantas de capim-marmelada e de soja, bem como o reflexo destas práticas nas relações de interferência entre a planta daninha e a planta cultivada.

\section{MATERIAL E MÉTODOS}

O trabalho foi instalado em casa-devegetação, pertencente ao Departamento de Pesquisa da CODETC, situado no município de Cascavel/PR. A terra utilizada como substrato de crescimento foi coletada na camada arável de um Latossolo Roxo distrófico. O substrato foi peneirado $\mathrm{m}$ Tamis $2 \mathrm{~mm}$ e, apresentava as seguintes características: M.O. $=3,3 \% ; \mathrm{pH}\left(\mathrm{CaCl}_{2}\right)$ $=4,4 ; \mathrm{P}(\mu \mathrm{g} / \mathrm{ml})=11 ; \mathrm{em}$ meq $/ 100 \mathrm{ml}$ de solo $(\mathrm{K}$ $=0,09, \mathrm{Ca}=3,40, \mathrm{Mg}=1,20, \mathrm{H}+\mathrm{Al}=7,20, \mathrm{Al}=$ $0,85, \mathrm{~S}=4,69)$ e V $(\%)=39$.

Utilizaram-se vasos plásticos com capacidade de 4 litros, com prato para evitar a perda de água após a irrigação, retornando sempre que necessário a água para o vaso. A irrigação foi diária e suficiente para a manutenção da umidade, sendo a irrigação escalonada durante o dia para evitar a percolação pelo fundo do recipiente.

Foi utilizada a variedade de Soja OCEPAR 4 - Iguaçu. Semeou-se seis sementes/vaso, a profundidade de $2 \mathrm{~cm}$, sendo que uma semana após, efetuou-se um desbaste, deixando três plantas/vaso (cotilêdones para fora do solo e extendidos-VC).

As sementes de capim-marmelada foram semeadas em uma bandeja plástica e quando as plântulas apresentavam duas folhas, 4 a $5 \mathrm{~cm}$ de altura, foram transplantadas, em número de 15 nos vasos (dia em que desbastou-se as plantas de soja) e, uma semana após, também sofreram desbaste, ficando 10 plantas/vaso. A temperatura da casade-vegetação foi mantida entre $28-29^{\circ} \mathrm{C}$ no período diurno e entre $19-20^{\circ} \mathrm{c}$ no período noturno.

$\mathrm{O}$ delineamento experimental adotado foi o inteiramente casualizado, com quatro repetições. Os tratamentos foram dispostos em um esquema fatorial $2 \times 2 \times 4$, onde constituíram as seguintes 
variáveis: dois níveis de calagem ( sem calagem e com a elevação da saturação de bases a 70\%, com $\mathrm{CaCO}_{3}$ ), três condições de vegetação nos vasos (três plantas de soja/vaso; dez plantas de capimmarmelada e três plantas de soja e dez plantas de capim-marmelada ) e quatro doses de aplicação de fósforo no substrato ( $0,50,100$ e $200 \mathrm{ppm}$ ), onde utilizou-se como fonte o fosfato monopotassico $\left(\mathrm{KH}_{2} \mathrm{PO}_{4}\right)$.

Foi utilizado o cloreto de potássio $(\mathrm{KCl})$ para corrigir os diferentes teores de potássio adicionados na adubação fosfatada. Com uma antecedência de 20 dias em relação a semeadura dos diásporos foi efetuada a calagem e a adubação do substrato, após o que, este foi mantido com umidade entre 50 e $70 \%$ do poder de emebebição, através de pesagens diárias. $\mathrm{O}$ experimento teve a duração de 49 dias (após o transplante das mudas de capim-marmelada).

Semanalmente, avaliou-se o comprimento da parte aérea das plantas de soja e de capimmarmelada, o número de folhas de ambas espécies, o número de perfihos da planta daninha $\mathrm{e}$, ao final do experimento, as plantas foram cortadas ao nível do solo, separando-se caule e folhas, sendo as folhas imediatamente fotocopiadas para realizar posteriormente a medição da área foliar. No caso da planta daninha denominou-se caule o conjunto caule + bainha.

Para retirada das raízes dos vasos, estes foram emersos em água e cuidadosamente processou-se. Todo material, parte aérea e radicular, foi lavado, seco em estufa de circulação forçada de ar $\left(65-70^{\circ} \mathrm{C}\right)$ e pesado. Para a avaliação dos teores de clorofila a e b utilizou-se a metodologia contida em Arnon (1949).

Os resultados obtidos foram submetidos a análise de variância pelo teste $\mathrm{F}$, segundo esquema fatorial $2 \times 3 \times 4$ e a comparação de médias foi efetuada pelo teste de Tukey ( $\mathrm{P}>0,05)$.

\section{RESULTADOS E DISCUSSÃO}

Na Tabela 1 estão apresentados os dados referentes aos efeitos da calagem, da convivência das plantas de capim-marmelada e da dose de fósforo sobre a altura média das plantas de soja e, na Tabela 2 os valores obtidos nos desdobramentos dos graus de liberdade dos tratamentos quando as interações foram significativas.

Os efeitos da calagem foram significativos desde os 21 dias após o início do estudo. A significância observada aos sete dias pode ser considerada aleatória, pois nesta ocasião, as plântulas de soja ainda utilizavam predominantemente as reservas dos cotilédones, sendo pouco influenciado pelas propriedades químicas do solo. A calagem incrementou a altura das plantas de soja entre 5,8 e 13,3\%, dependendo da época de avaliação. A interferência imposta pelo capim-marmelada passou a reduzir a altura de plantas de soja a partir dos 28 dias, variando de 6,6 a 13,4\% de acordo com o período estudado.

A interação dos efeitos da calagem e da presença da planta daninha sobre a altura das plantas de soja foram significativas aos 14 e 49 dias. Nestas duas épocas, pode-se observar que os efeitos da calagem apenas foram significativos nos tratamentos onde a soja cresceu isolada. A interferência da planta daninha anulou a resposta da leguminosa à calagem.

A adubação fosfatada influenciou a altura das plantas de soja, sendo que aos 49 dias as três doses de fósforo praticamente eqüivaleram-se em termos de influência sobre a altura da soja. A interação entre calagem e doses de fósforo foi significativa nas avaliações de 14,21 e 49 dias após o início do estudo. No final do estudo, notase que a calagem incrementou a altura de plantas de soja apenas nas parcelas que receberam adubação com fósforo, independente da dose.

As respostas da soja a calagem e fósforo eram esperadas, pois a acidez e os teores de fósforo originais do solo são considerados baixos e, os teores de $\mathrm{Al}$ altos, de acordo com as exigências das plantas de soja ( Raij et al., 1977; Camargo et al., 1982; Nakayama, et al., $1984 \mathrm{e}$ Samarrão et al., 1986). Os efeito negativos da convivência da soja com plantas daninhas já foram observados também por outros pesquisadores, como (Leguizamon et al., 1985 e Wulff, 1987). 
TABELA 1. Valores médios verificados para altura de plantas de soja em diferentes épocas de avaliação obtidos no desdobramento dos graus de liberdade das variáveis principais.

\begin{tabular}{|c|c|c|c|c|c|c|c|}
\hline \multirow{2}{*}{ Condição } & \multicolumn{7}{|c|}{ Altura de plantas $(\mathrm{cm})$ - DAIE } \\
\hline & 07 & 14 & 21 & 28 & 35 & 42 & 49 \\
\hline & \multicolumn{7}{|c|}{ Efeito da Calagem (CA) } \\
\hline Com & $10,80 \mathrm{a}^{1}$ & 15,26 & $21,57 \mathrm{a}$ & $27,60 \mathrm{a}$ & $33,59 a$ & $38,47 \mathrm{a}$ & $47,10 \mathrm{a}$ \\
\hline \multirow[t]{2}{*}{ Sem } & $10,21 \mathrm{~b}$ & 14,80 & $20,47 \mathrm{~b}$ & $25,62 \mathrm{~b}$ & $29,99 \mathrm{~b}$ & $35,65 \mathrm{~b}$ & $41,56 \mathrm{~b}$ \\
\hline & \multicolumn{7}{|c|}{ Efeito da Convivência $(\mathrm{CV})$} \\
\hline Sem & 10,61 & 15,31 & 21,16 & $27,51 \mathrm{a}$ & $33,90 \mathrm{a}$ & $39,72 \mathrm{a}$ & $46,55 \mathrm{a}$ \\
\hline \multirow[t]{2}{*}{ Com } & 10,40 & 14,75 & 20,87 & $25,70 \mathrm{~b}$ & $29,68 \mathrm{~b}$ & $34,41 \mathrm{~b}$ & $42,11 \mathrm{~b}$ \\
\hline & \multicolumn{7}{|c|}{ Efeito da dose de Fósforo (DF) } \\
\hline$(\mathrm{ppm})$ & & & & & & & \\
\hline 00 & $10,04 b$ & $11,92 \mathrm{c}$ & $16,19 \mathrm{c}$ & $18,27 \mathrm{c}$ & $22,51 b$ & $27,09 \mathrm{c}$ & $34,24 b$ \\
\hline 50 & $10,32 \mathrm{ab}$ & $14,07 b$ & $20,76 b$ & $28,04 \mathrm{~b}$ & $34,22^{\mathrm{a}}$ & $39,41 b$ & $46,68 \mathrm{a}$ \\
\hline 100 & $10,29 \mathrm{ab}$ & $15,21 \mathrm{~b}$ & $22,27 b$ & $29,02 b$ & $34,71^{\mathrm{a}}$ & $39,66 \mathrm{ab}$ & $47,43 \mathrm{a}$ \\
\hline 200 & $11,04 \mathrm{a}$ & $18,92 \mathrm{a}$ & $24,84 a$ & $31,10^{\mathrm{a}}$ & $35,72^{\mathrm{a}}$ & $42,09 \mathrm{a}$ & $48,97 \mathrm{a}$ \\
\hline c.v. $(\%)$ & 5,09 & 7,01 & 6,04 & 8,09 & 7,07 & 9,04 & 9,01 \\
\hline d.m.s. (CA e CV) & 0,48 & 0,71 & 0,82 & 1,20 & 1,03 & 1,32 & 1,32 \\
\hline d.m.s. (DF) & 0,90 & 1,34 & 1,53 & 2,24 & 1,92 & 2,46 & 2,48 \\
\hline
\end{tabular}

TABELA 2. Valores médios para a altura de plantas de soja em diferentes épocas de avaliação obtidos no desdobramento dos graus de liberdade das interações entre calagem e convivência e, calagem e dose de fósforo.

\begin{tabular}{|c|c|c|c|c|c|c|}
\hline \multirow{2}{*}{ Convivência } & \multicolumn{6}{|c|}{ Altura de plantas $(\mathrm{cm})$ - DAIE } \\
\hline & \multicolumn{2}{|c|}{14} & \multicolumn{2}{|c|}{21} & \multicolumn{2}{|c|}{49} \\
\hline & \multicolumn{2}{|c|}{ Convivência } & \multicolumn{2}{|c|}{ Convivência } & \multicolumn{2}{|c|}{ Convivência } \\
\hline Calagem & Sem & Com & Sem & Com & Sem & Com \\
\hline Com & A15,90a & B14,62 & - & - & A50,12a & B 44,04 \\
\hline Sem & $14,87 b$ & 14,71 & - & - & A42,96b & $\mathrm{B} 40,17$ \\
\hline \multirow[t]{2}{*}{ d.m.s } & \multicolumn{2}{|c|}{1,01} & \multicolumn{2}{|c|}{-} & \multicolumn{2}{|c|}{1,87} \\
\hline & \multicolumn{2}{|c|}{ Calagem } & \multicolumn{2}{|c|}{ Calagem } & \multicolumn{2}{|c|}{ Calagem } \\
\hline \multicolumn{7}{|l|}{$(\mathrm{ppm})$} \\
\hline 00 & $\mathrm{~A} 13,09 \mathrm{~b}$ & $\mathrm{~B} 10,75 \mathrm{~d}$ & A $17,52 b$ & $\mathrm{~B} 14,86 \mathrm{~b}$ & $33,87 b$ & $34,60 \mathrm{~b}$ \\
\hline 50 & $14,75 b$ & $13,39 \mathrm{c}$ & A $21,90 a$ & B19,62a & A50,71a & B42,04a \\
\hline 100 & $14,97 b$ & $15,45 b$ & $23,09 \mathrm{a}$ & $21,42 \mathrm{a}$ & A51,32a & B44,15a \\
\hline 200 & $18,24 \mathrm{a}$ & $19,60 \mathrm{a}$ & $23,75 \mathrm{a}$ & $25,94 a$ & A52,49a & B45,47a \\
\hline d.m.s (coluna) & \multicolumn{2}{|c|}{1,89} & \multicolumn{2}{|c|}{2,17} & \multicolumn{2}{|c|}{3,50} \\
\hline d.m.s (linha) & \multicolumn{2}{|c|}{2,65} & \multicolumn{2}{|c|}{1,64} & \multicolumn{2}{|c|}{1,64} \\
\hline
\end{tabular}

${ }^{1}$ Médias seguidas de mesma letra, minúscula na coluna e maiúscula na linha, não diferem estatisticamente entre si pelo teste de tukey (P>0,05). DAIE - Dias após o início do experimento. 
Na Tabela 3 estão apresentados os dados referentes aos efeitos da calagem, da convivência das plantas de soja e da dose de fósforo sobre a altura média das plantas de $B$. plantaginea e, na Tabela 4, os valores obtidos nos desdobramentos dos graus de liberdade dos tratamentos quando as interações foram significativas.

Os efeitos da calagem foram significativos desde os 14 dias após o início do estudo. A calagem proporcionou reduções entre 6,7 e 15,0\% na altura das plantas de capim-marmelada, dependendo do período avaliado. A interferência imposta pela soja reduziu a altura da planta daninha a partir dos 35 dias, com valores entre 6,8 e 7,8\%, dependendo da época analisada. Estes resultados constituem um forte indicativo da adaptação desta planta daninha a solos ácidos, explicando talvez, a sua grande importância na região Sul e Central do Brasil

$\mathrm{O}$ uso de adubo fosfatado incrementou a altura de plantas de $B$. plantaginea desde o início do experimento. A partir dos 35 dias não se observaram mais diferenças entre três doses de fósforo aplicadas (50, 100 e 200 ppm). Os incrementos observados na altura da planta daninha com a aplicação de $200 \mathrm{ppm}$ de $\mathrm{P}$ variaram de 17,3 a $82,1 \%$, de acordo com o período estudado. A diferença entre doses apenas influenciou a velocidade de manifestação do efeito e, no final do estudo não houve diferenças entre as doses aplicadas.

A interação entre calagem e doses de fósforo foi significativa em vário períodos. Ao final do estudo, aos 49 dias, a calagem proporcionou decréscimos na altura de plantas de $B$. plantaginea apenas nas parcelas que receberam 200 ppm. Quando se compara os efeitos das doses de $\mathrm{P}$ dentro dos grupos de tratamentos com e sem calagem, observa-se ao final do experimento, que independentemente da dose de $\mathrm{P}$, houve incrementos na altura das plantas de $B$. plantaginea.

A interação entre presença de soja e doses de fósforo foi significativa apenas nas avaliações efetuadas aos 42 e 49 dias, sendo que em ambas, verifica-se que a interferência imposta pela soja reduziu a altura da planta daninha apenas no grupo de parcelas que receberam 50 e 100 ppm de P. A aplicação de $\mathrm{P}$, independentemente da dose, incrementou a altura das plantas tanto no grupo de parcelas com e sem convivência.

Na Tabela 5, estão apresentados os dados referentes aos efeitos da calagem, da convivência das plantas de soja e da dose de fósforo sobre o número de trifólios de soja e, na Tabela 6 , os valores obtidos nos desdobramentos dos graus de liberdade dos tratamentos quando as interações foram significativas.

De um modo geral, a calagem aumentou significativamente o número de trifólios de soja, com incrementos da ordem de 4,4 a 10,0\%, de acordo com a época avaliada e, a interferência imposta pela $B$. plantaginea determinou reduções entre 4,6 e 9,7\% no número de trifólios de soja, a partir dos 28 dias, como observado por (Nakayama et al., 1984 e Samarrão et al, 1986).

A adubação fosfatada influenciou o aparecimento de trifólios na planta de soja e, em todas as épocas estudadas, a aplicação de fósforo incrementou o seu número, sendo que a aplicação de $200 \mathrm{ppm}$ determinou incrementos que variaram entre 10,2 e $34,5 \%$, dependendo da época analisada.

A interação entre calagem e doses de fósforo foi significativa já a partir dos 21 dias. Ao final do estudo, a calagem incrementou o número de trifólios apenas nas parcelas que receberam 50 e 100 ppm de P. Ao comparar-se a aplicação do fósforo dentro de cada grupo de tratamento, sem e com calagem, nota-se que apenas $50 \mathrm{ppm}$ de $\mathrm{P}$ foi suficiente para aumentar o número de trifólios de soja, independente da época avaliada. A simples resposta à aplicação de fósforo refletiu os baixos teores naturais do mesmo no solo (Raij \& Quaggio, 1983), mas a resposta constante às doses crescente do elemento sugerem ser a soja ou esta variedade uma planta exigente em fósforo.

A interação entre presença de $B$. plantaginea e as doses de fósforo foi significativa aos 35 e 49 dias. Em ambas épocas, a planta daninha determinou decréscimos no número de trifólios de soja em todos os grupos de parcelas que receberam a adubação fosfatada. 
TABELA 3. Valores médios verificados para altura de plantas de Brachiaria plantaginea em diferentes épocas de avaliação obtidos no desdobramento dos graus de liberdade das variáveis principais.

\begin{tabular}{|c|c|c|c|c|c|c|c|}
\hline \multirow{2}{*}{ Condição } & \multicolumn{7}{|c|}{ Altura de plantas $(\mathrm{cm})$ - DAIE } \\
\hline & 07 & 14 & 21 & 28 & 35 & 42 & 49 \\
\hline & \multicolumn{7}{|c|}{ Efeito da Calagem (CA) } \\
\hline Com & 5,16 & $9,23 \mathrm{~b}^{1}$ & $15,44 b$ & $22,26 \mathrm{~b}$ & $26,22 b$ & $29,67 b$ & 37,19 \\
\hline \multirow[t]{2}{*}{ Sem } & 5,31 & $10,86 \mathrm{a}$ & $18,00 \mathrm{a}$ & $24,73 \mathrm{a}$ & $28,97 \mathrm{a}$ & $31,83 \mathrm{a}$ & 36,18 \\
\hline & \multicolumn{7}{|c|}{ Efeito da Convivência (CV) } \\
\hline Sem & 5,14 & 10,07 & 16,54 & 24,01 & $28,61 \mathrm{a}$ & $31,83 \mathrm{a}$ & $38,17 \mathrm{a}$ \\
\hline \multirow[t]{2}{*}{ Com } & 5,33 & 10,02 & 16,92 & 22,98 & $26,58 \mathrm{~b}$ & $29,67 \mathrm{~b}$ & $35,19 \mathrm{~b}$ \\
\hline & \multicolumn{7}{|c|}{ Efeito da dose de Fósforo (DF) } \\
\hline (ppm) & & & & & & & \\
\hline 00 & $4,78 \mathrm{c}$ & $4,92 \mathrm{~d}$ & $5,06 \mathrm{c}$ & $5,52 \mathrm{c}$ & $7,75 b$ & $12,79 \mathrm{~b}$ & $19,96 \mathrm{~b}$ \\
\hline 50 & $5,04 \mathrm{bc}$ & $9,04 \mathrm{c}$ & $17,43 b$ & $27,32 \mathrm{~b}$ & $33,46 \mathrm{a}$ & $36,37 \mathrm{a}$ & $43,62 \mathrm{a}$ \\
\hline 100 & $5,34 \mathrm{~b}$ & $12,05 \mathrm{~b}$ & $21,71 \mathrm{a}$ & $30,32 \mathrm{a}$ & $34,02 \mathrm{a}$ & $37,50 \mathrm{a}$ & $40,92 \mathrm{a}$ \\
\hline 200 & $5,78 \mathrm{a}$ & $14,16 \mathrm{a}$ & $22,73 \mathrm{a}$ & $30,81 \mathrm{a}$ & $35,16 \mathrm{a}$ & $36,35 \mathrm{a}$ & $40,24 \mathrm{a}$ \\
\hline c.v. $(\%)$ & 8,08 & 13,02 & 10,07 & 10,8 & 12,01 & 11,00 & 11,08 \\
\hline d.m.s. (CA e CV) & 0,23 & 0,66 & 0,89 & 1,28 & 1,75 & 1,71 & 2,18 \\
\hline d.m.s. (DF) & 0,43 & 1,24 & 1,68 & 2,40 & 3,27 & 3,19 & 4,08 \\
\hline
\end{tabular}

${ }^{1}$ Médias seguidas de mesma letra na coluna não diferem estatisticamente entre si pelo teste de tukey $(\mathrm{P}>0,05)$.

DAIE - Dias após o início do experimento.

TABELA 4. Valores médios verificados para a altura de plantas de Brachiaria plantaginea em diferentes épocas de avaliação obtidos no desdobramento dos graus de liberdade das interações significativas entre calagem e dose de fósforo e, convivência e dose de fósforo.

\begin{tabular}{|c|c|c|c|c|c|c|c|c|c|c|}
\hline \multirow[t]{2}{*}{ Interação } & \multicolumn{10}{|c|}{ Altura de plantas $(\mathrm{cm})$ - DAIE } \\
\hline & \multicolumn{2}{|c|}{14} & \multicolumn{2}{|c|}{21} & \multicolumn{2}{|c|}{35} & \multicolumn{2}{|c|}{42} & \multicolumn{2}{|c|}{49} \\
\hline & \multicolumn{2}{|c|}{ Calagem } & \multicolumn{2}{|c|}{ Calagem } & \multicolumn{2}{|c|}{ Calagem } & \multicolumn{2}{|c|}{ Calagem } & \multicolumn{2}{|c|}{ Calagem } \\
\hline $\begin{array}{l}\text { Dose de } \\
\text { Fósforo } \\
\text { (ppm) }\end{array}$ & Com & Sem & Com & Sem & Com & Sem & Com & Sem & Com & Sem \\
\hline 00 & $4,96 \mathrm{~d}$ & $4,95 \mathrm{c}$ & $5,05 \mathrm{c}$ & $5,06 \mathrm{~b}$ & $7,80 \mathrm{~b}$ & $7,70 \mathrm{~b}$ & - & - & $18,25 \mathrm{c}$ & $21,66 b$ \\
\hline 50 & $\mathrm{~B} 08,19 \mathrm{c}$ & $9,88 \mathrm{~b}$ & $\mathrm{~B} 16,34 \mathrm{~b}$ & $\mathrm{~A} 18,52 \mathrm{a}$ & B31,57a & $\mathrm{A} 35,34 \mathrm{a}$ & - & - & $44,39 \mathrm{a}$ & $42,86 a$ \\
\hline 100 & $\mathrm{~B} 10,57 \mathrm{~b}$ & $\mathrm{~A} 13,52 \mathrm{a}$ & $\mathrm{B} 19,42 \mathrm{a}$ & $\mathrm{A} 24,00 \mathrm{a}$ & $34,92 \mathrm{a}$ & $35,89 a$ & - & - & $44,29 \mathrm{a}$ & $41,55 a$ \\
\hline 200 & B13,25a & A15,06a & B20,96 a & $\mathrm{A} 24,50 \mathrm{a}$ & B30,60a & A $37,45 a$ & - & - & B37,79b & A42,69a \\
\hline $\begin{array}{l}\text { d.m.s } \\
\text { (coluna) }\end{array}$ & \multicolumn{2}{|c|}{1,76} & \multicolumn{2}{|c|}{2,38} & \multicolumn{2}{|c|}{4,63} & \multicolumn{2}{|c|}{ - } & \multicolumn{2}{|c|}{5,37} \\
\hline $\begin{array}{l}\text { d.m.s } \\
\text { (linha) }\end{array}$ & \multicolumn{2}{|c|}{1,33} & \multicolumn{2}{|c|}{1,80} & \multicolumn{2}{|c|}{3,50} & \multicolumn{2}{|c|}{-} & \multicolumn{2}{|c|}{4,37} \\
\hline & \multicolumn{2}{|c|}{ Convivência } & \multicolumn{2}{|c|}{ Convivência } & \multicolumn{2}{|c|}{ Convivência } & \multicolumn{2}{|c|}{ Convivência } & \multicolumn{2}{|c|}{ Convivência } \\
\hline $\begin{array}{l}\text { Dose de } \\
\text { Fósforo } \\
(\mathrm{ppm})\end{array}$ & Sem & Com & Sem & Com & Sem & Com & Sem & Com & Sem & Com \\
\hline 00 & - & - & - & - & - & - & $11,74 b$ & $13,84 b$ & $18,29 \mathrm{c}$ & $21,62 b$ \\
\hline 50 & - & - & - & - & - & - & A $38,95 a$ & $\mathrm{~B} 33,80 \mathrm{a}$ & A46,65a & B $40,60 a$ \\
\hline 100 & - & - & - & - & - & - & $\mathrm{A} 40,42 \mathrm{a}$ & B34,57a & $\mathrm{A} 47,20 \mathrm{a}$ & B38,64a \\
\hline 200 & - & - & - & - & - & - & $36,21 \mathrm{a}$ & $36,49 \mathrm{a}$ & $40,56 b$ & $39,91 \mathrm{a}$ \\
\hline $\begin{array}{l}\text { d.m.s } \\
\text { (coluna) }\end{array}$ & \multicolumn{2}{|c|}{-} & & & \multicolumn{2}{|c|}{-} & \multicolumn{2}{|c|}{4,52} & \multicolumn{2}{|c|}{5,77} \\
\hline $\begin{array}{l}\text { d.m.s } \\
\text { (linha) }\end{array}$ & \multicolumn{2}{|c|}{-} & & & \multicolumn{2}{|c|}{-} & \multicolumn{2}{|c|}{3,41} & \multicolumn{2}{|c|}{4,36} \\
\hline
\end{tabular}

${ }^{1}$ Médias seguidas de mesma letra, minúscula na coluna e maiúscula na linha, não diferem estatisticamente entre si pelo teste de Tukey ( $\left.\mathrm{P}>0,05\right)$.

DAIE - Dias após o início do experimento. 
TABELA 5. Valores médios verificados para o número de trifólios de soja por vaso, transformados em $\sqrt{x}$, em diferentes épocas de avaliação obtidos no desdobramento dos graus de liberdade das variáveis principais.

\begin{tabular}{|c|c|c|c|c|c|c|c|}
\hline \multirow{2}{*}{ Condição } & \multicolumn{7}{|c|}{ Número de trifólios - DAIE } \\
\hline & 07 & 14 & 21 & 28 & 35 & 42 & 49 \\
\hline & \multicolumn{7}{|c|}{ Efeito da Calagem (CA) } \\
\hline Com & - & 1,74 & $2,28 \mathrm{a}^{1}$ & 2,79 & $3,29 \mathrm{a}$ & $3,71 \mathrm{a}$ & $5,63 \mathrm{a}$ \\
\hline \multirow[t]{2}{*}{ Sem } & - & 1,73 & $2,15 \mathrm{~b}$ & 2,73 & $3,15 b$ & $3,53 \mathrm{~b}$ & $5,12 b$ \\
\hline & \multicolumn{7}{|c|}{ Efeito da Convivência (CV) } \\
\hline Sem & - & 1,73 & 2,23 & $2,82 \mathrm{a}$ & $3,30 \mathrm{a}$ & $4,74 \mathrm{a}$ & $5,65 \mathrm{a}$ \\
\hline \multirow[t]{2}{*}{ Com } & - & 1,74 & 2,20 & $2,29 \mathrm{~b}$ & $3,13 b$ & $3,50 \mathrm{~b}$ & $5,10 \mathrm{~b}$ \\
\hline & \multicolumn{7}{|c|}{ Efeito da dose de Fósforo (DF) } \\
\hline$(\mathrm{ppm})$ & & & & & & & \\
\hline 00 & - & $1,66 b$ & $1,84 \mathrm{c}$ & $2,39 \mathrm{c}$ & $2,64 \mathrm{c}$ & $3,08 \mathrm{c}$ & $4,41 \mathrm{~b}$ \\
\hline 50 & - & $1,73 \mathrm{ab}$ & $2,21 \mathrm{ab}$ & $2,74 b$ & $3,31 b$ & $3,72 \mathrm{ab}$ & $5,60 \mathrm{a}$ \\
\hline 100 & - & $1,71 \mathrm{ab}$ & $2,34 \mathrm{ab}$ & $2,81 \mathrm{~b}$ & $3,38 b$ & $3,79 \mathrm{a}$ & $5,75 a$ \\
\hline 200 & - & $1,83 a$ & $2,47 a$ & $3,09 \mathrm{a}$ & $3,54 \mathrm{a}$ & $3,90 \mathrm{a}$ & $5,74 \mathrm{a}$ \\
\hline c.v.(\%) & - & 9,02 & 7,07 & 7,02 & 5,00 & 4,03 & 6,09 \\
\hline d.m.s. (CA e CV) & - & 0,08 & 0,09 & 0,10 & 0,08 & 0,08 & 0,19 \\
\hline d.m.s. (DF) & - & 0,15 & 0,16 & 0,19 & 0,15 & 0,15 & 0,35 \\
\hline
\end{tabular}

${ }^{1}$ Médias seguidas de mesma letra na coluna não diferem estatisticamente entre si pelo teste de tukey $(\mathrm{P}>0,05)$.

DAIE - Dias após o início do experimento.

TABELA 6. Valores médios verificados para o número de trifólios de soja por vaso, transformados em $\sqrt{x}$, em diferentes épocas de avaliação obtidos no desdobramento dos graus de liberdade das interações significativas entre calagem e dose de fósforo e, convivência e dose de fósforo.

\begin{tabular}{|c|c|c|c|c|c|c|c|c|c|c|}
\hline \multirow[t]{2}{*}{ Interação } & \multicolumn{10}{|c|}{ Número de trifólios - DAIE } \\
\hline & \multicolumn{2}{|c|}{21} & \multicolumn{2}{|c|}{28} & \multicolumn{2}{|c|}{35} & \multicolumn{2}{|c|}{42} & \multicolumn{2}{|c|}{49} \\
\hline & \multicolumn{2}{|c|}{ Calagem } & \multicolumn{2}{|c|}{ Calagem } & \multicolumn{2}{|c|}{ Calagem } & \multicolumn{2}{|c|}{ Calagem } & \multicolumn{2}{|c|}{ Calagem } \\
\hline $\begin{array}{l}\text { Dose de } \\
\text { Fósforo } \\
(\mathrm{ppm})\end{array}$ & Com & Sem & Com & Sem & Com & Sem & Com & Sem & Com & Sem \\
\hline 00 & $\mathrm{~A} 1,96 \mathrm{~b}$ & $\mathrm{~B} 1,73 \mathrm{c}$ & $2,39 b$ & $2,39 \mathrm{c}$ & $2,67 b$ & $2,61 \mathrm{c}$ & $3,08 \mathrm{~b}$ & $3,08 \mathrm{c}$ & $4,23 b$ & $4,59 \mathrm{~b}$ \\
\hline 50 & $\mathrm{~A} 2,37 \mathrm{a}$ & $\mathrm{B} 2,05 \mathrm{~b}$ & $\mathrm{~A} 2,91 \mathrm{a}$ & $\mathrm{B} 2,57 \mathrm{~b}$ & A3,48a & B3, $14 b$ & A3,90a & B3, 54b & $\mathrm{A} 6,12 \mathrm{a}$ & B5,09a \\
\hline 100 & $2,37 \mathrm{a}$ & $2,82 \mathrm{a}$ & $2,82 \mathrm{a}$ & $2,80 \mathrm{~b}$ & $3,44 \mathrm{a}$ & $3,31 \mathrm{~b}$ & A $3,87 \mathrm{a}$ & $\mathrm{B} 3,70 \mathrm{ab}$ & A6,24a & $\mathrm{B} 5,26 \mathrm{a}$ \\
\hline 200 & $2,42 a$ & $2,52 \mathrm{a}$ & $3,03 \mathrm{a}$ & $3,15 \mathrm{a}$ & $3,56 a$ & $3,53 \mathrm{a}$ & A3,98a & $\mathrm{B} 3,82 \mathrm{a}$ & $5,92 \mathrm{a}$ & $5,59 \mathrm{a}$ \\
\hline d.m.s $s^{2}$ & \multicolumn{2}{|c|}{0,23} & \multicolumn{2}{|c|}{0,26} & \multicolumn{2}{|c|}{0,22} & \multicolumn{2}{|c|}{0,26} & \multicolumn{2}{|c|}{0,50} \\
\hline d.m.s $s^{3}$ & \multicolumn{2}{|c|}{0,17} & \multicolumn{2}{|c|}{0,20} & \multicolumn{2}{|c|}{0,16} & \multicolumn{2}{|c|}{0,16} & \multicolumn{2}{|c|}{0,37} \\
\hline & \multicolumn{2}{|c|}{ Convivência } & \multicolumn{2}{|c|}{ Convivência } & \multicolumn{2}{|c|}{ Convivência } & \multicolumn{2}{|c|}{ Convivência } & \multicolumn{2}{|c|}{ Convivência } \\
\hline $\begin{array}{l}\text { Dose de } \\
\text { Fósforo } \\
(\mathrm{ppm})\end{array}$ & Sem & Com & Sem & Com & Sem & Com & Sem & Com & Sem & Com \\
\hline 00 & - & - & - & - & $2,62 \mathrm{c}$ & $2,66 \mathrm{~b}$ & - & - & 4,40b & $4,42 b$ \\
\hline 50 & - & - & - & - & $\mathrm{A} 3,40 \mathrm{~b}$ & $\mathrm{~B} 3,21 \mathrm{a}$ & - & - & A6,09a & B5,11a \\
\hline 100 & - & - & - & - & A $3,48 b$ & $\mathrm{~B} 3,27 \mathrm{a}$ & - & - & A6,05a & B5,44a \\
\hline 200 & - & - & - & - & $\mathrm{A} 3,27 \mathrm{a}$ & B3,37a & - & - & $\mathrm{A} 6,07 \mathrm{a}$ & $\mathrm{B} 5,42 \mathrm{a}$ \\
\hline d.m.s $s^{2}$ & \multirow{2}{*}{\multicolumn{2}{|c|}{-}} & \multirow{2}{*}{\multicolumn{2}{|c|}{-}} & \multicolumn{2}{|c|}{0,22} & \multicolumn{2}{|c|}{ - } & \multicolumn{2}{|c|}{0,49} \\
\hline d.m.s ${ }^{3}$ & & & & & \multicolumn{2}{|c|}{0,16} & & & & \\
\hline
\end{tabular}


Na Tabela 7, estão apresentados os plantaginea e, na Tabela 8, os valores obtidos no dados referentes aos efeitos da calagem, da convivência das plantas de soja e da dose de fósforo sobre o número de perfilhos de Brachiaria desdobramento dos graus de liberdade dos tratamentos quando as interações foram significativas.

TABELA 7. Valores médios verificados para o número de perfilhos de Brachiaria plantaginea por vaso, transformados em $\sqrt{x+1}$, em diferentes épocas de avaliação obtidos no desdobramento dos graus de liberdade das variáveis principais.

\begin{tabular}{|c|c|c|c|c|c|c|c|}
\hline \multirow{2}{*}{ Condição } & \multicolumn{7}{|c|}{ Número de perfilhos - DAIE } \\
\hline & 07 & 14 & 21 & 28 & 35 & 42 & 49 \\
\hline & \multicolumn{7}{|c|}{ Efeito da Calagem (CA) } \\
\hline Com & - & $1,07 \mathrm{~b}$ & $1,98 b^{1}$ & $2,56 \mathrm{~b}$ & $2,69 \mathrm{~b}$ & $2,69 \mathrm{~b}$ & $2,71 b$ \\
\hline \multirow[t]{2}{*}{ Sem } & - & $1,51 \mathrm{a}$ & $2,42 \mathrm{a}$ & $2,92 \mathrm{a}$ & $3,03^{\mathrm{a}}$ & $3,03 \mathrm{a}$ & $2,98 \mathrm{a}$ \\
\hline & \multicolumn{7}{|c|}{ Efeito da Convivência (CV) } \\
\hline Sem & - & $1,50 \mathrm{a}$ & $2,77 \mathrm{a}$ & $3,38 \mathrm{a}$ & $3,46^{\mathrm{a}}$ & $3,46 \mathrm{a}$ & $3,49 \mathrm{a}$ \\
\hline Com & - & $1,08 \mathrm{~b}$ & $1,64 \mathrm{~b}$ & $2,10 \mathrm{~b}$ & $2,25 b$ & $2,25 b$ & $2,19 \mathrm{~b}$ \\
\hline \multirow{2}{*}{\multicolumn{8}{|c|}{ Efeito da dose de Fósforo (DF) }} \\
\hline & & & & & & & \\
\hline 00 & - & $1,00 \mathrm{~b}$ & $1,00 \mathrm{~d}$ & $1,00 \mathrm{c}$ & $1,00 \mathrm{c}$ & $1,00 \mathrm{c}$ & $1,10 \mathrm{~b}$ \\
\hline 50 & - & $1,05 \mathrm{~b}$ & $2,14 \mathrm{ac}$ & $3,03 b$ & $3,31 b$ & $3,30 \mathrm{~b}$ & $3,30 \mathrm{a}$ \\
\hline 100 & - & $1,47 \mathrm{a}$ & $2,58 \mathrm{~b}$ & $3,36 \mathrm{a}$ & $3,44 \mathrm{ab}$ & $3,44 \mathrm{ab}$ & $3,40 \mathrm{a}$ \\
\hline 200 & - & $1,64 \mathrm{a}$ & $3,10 \mathrm{a}$ & $3,56 \mathrm{a}$ & $3,69^{\mathrm{a}}$ & $3,69 \mathrm{a}$ & $3,56 \mathrm{a}$ \\
\hline c.v. $(\%)$ & - & 19,09 & 14,01 & 12,03 & 10,04 & 11,06 & 12,02 \\
\hline d.m.s. (CA e CV) & - & 0,13 & 0,16 & 0,17 & 0,15 & 0,16 & 0,17 \\
\hline d.m.s. (DF) & - & 0,24 & 0,29 & 0,32 & 0,28 & 0,30 & 0,33 \\
\hline
\end{tabular}

${ }^{1}$ Médias seguidas de mesma letra na coluna não diferem estatisticamente entre si pelo teste de tukey $(\mathrm{P}>0,05)$.

DAIE - Dias após o início do experimento.

O efeito da calagem sobre o número de perfilhos de B. plantaginea foram deletéricos a partir dos 14 dias, com decréscimos variando entre 9,1 e $29,1 \%$, dependendo do período avaliado. A interferência imposta pela soja passou a reduzir o número de perfilhos a partir dos 14 dias, com valores entre 28,0 e $40,8 \%$, dependendo da época analisada.

A interação entre calagem e a presença de soja sobre o número de perfilhos de $B$. plantaginea mostrou-se significativa apenas aos 14 dias, onde verifica-se que a interferência da soja anulou as respostas negativas do capim-marmelada à calagem, pois foi o fator ecológico mais importante.
O uso de adubo fosfatado aumentou o número de perfilhos da planta daninha, na maioria das épocas estudadas, já com a aplicação de 50 ppm de P. A aplicação de $200 \mathrm{ppm}$ de P determinou incrementos entre 64,0 e $269,0 \%$, dependendo da época estudada. Na interação entre calagem e doses de fósforo observa-se que a calagem passou a ser o fator limitante quando houve a aplicação de fósforo.

$\mathrm{Na}$ interação entre presença de soja e as doses de fósforo, observa-se que a interferência imposta pela soja reduziu o número de perfilhos de capim-marmelada apenas no grupo de parcelas que receberam fósforo, exceto nas que receberam 50 ppm aos 14 dias. 
TABELA 8. Valores médios verificados para o número de perfilhos de Brachiaria plantaginea por vaso, transformados em $\sqrt{x+1}$, em diferentes épocas de avaliação obtidos no desdobramento dos graus de liberdade das interações entre calagem e convivência, calagem e dose de fósforo e, convivência e dose de fósforo.

\begin{tabular}{|c|c|c|c|c|c|c|c|c|c|c|c|c|}
\hline \multirow{2}{*}{ Interação } & \multicolumn{12}{|c|}{ Altura de plantas $(\mathrm{cm})$ - DAIE } \\
\hline & \multicolumn{2}{|c|}{14} & \multicolumn{2}{|c|}{21} & \multicolumn{2}{|c|}{28} & \multicolumn{2}{|c|}{35} & \multicolumn{2}{|c|}{42} & \multicolumn{2}{|c|}{49} \\
\hline & \multicolumn{2}{|c|}{ Convivência } & \multicolumn{2}{|c|}{ Convivência } & \multicolumn{2}{|c|}{ Convivência } & \multicolumn{2}{|c|}{ Convivência } & \multicolumn{2}{|c|}{ Convivência } & \multicolumn{2}{|c|}{ Convivência } \\
\hline Calagem & Sem & Com & Sem & Com & Sem & Com & Sem & Com & Sem & Com & Sem & Com \\
\hline Com & $1,08 \mathrm{~b}$ & 1,06 & - & - & - & - & - & - & - & - & - & - \\
\hline Sem & $\mathrm{A} 1,93^{\mathrm{a}}$ & $\mathrm{B} 1,09$ & - & - & - & - & - & - & - & - & - & - \\
\hline \multirow[t]{2}{*}{ d.m.s } & \multicolumn{2}{|c|}{0,18} & \multicolumn{2}{|c|}{ - } & \multicolumn{2}{|c|}{ - } & \multicolumn{2}{|c|}{ - } & \multicolumn{2}{|c|}{-} & \multicolumn{2}{|c|}{ - } \\
\hline & \multicolumn{2}{|c|}{ Calagem } & \multicolumn{2}{|c|}{ Calagem } & \multicolumn{2}{|c|}{ Calagem } & \multicolumn{2}{|c|}{ Calagem } & \multicolumn{2}{|c|}{ Calagem } & \multicolumn{2}{|c|}{ Calagem } \\
\hline $\begin{array}{l}\text { Dose de } \\
\text { Fósforo } \\
\text { (ppm) }\end{array}$ & Com & Sem & Com & Sem & Com & Sem & Com & Sem & Com & Sem & Com & Sem \\
\hline 00 & 1,00 & $1,00 \mathrm{~b}$ & $1,00 \mathrm{c}$ & $1,00 \mathrm{c}$ & $1,00 \mathrm{c}$ & $1,00 \mathrm{~b}$ & $1,00 \mathrm{c}$ & $1,00 \mathrm{~b}$ & $1,00 \mathrm{c}$ & $1,00 \mathrm{~b}$ & $1,15 \mathrm{c}$ & $1,05 b$ \\
\hline 50 & 1,00 & $1,09 b$ & $\mathrm{~B} 1,87 \mathrm{~b}$ & $\mathrm{~A} 2,42 \mathrm{~b}$ & $\mathrm{~B} 2,66 \mathrm{~b}$ & A3,41a & $\mathrm{B} 2,93 \mathrm{~b}$ & A3,69a & $\mathrm{B} 2,93 \mathrm{~b}$ & A3,66a & $\mathrm{B} 2,96 \mathrm{~b}$ & $\mathrm{~A} 3,65 \mathrm{a}$ \\
\hline 100 & $\mathrm{~B} 1,05$ & $\mathrm{~A} 1,89 \mathrm{a}$ & $\mathrm{B} 2,09 \mathrm{~b}$ & A3,06a & $\mathrm{B} 03,07 \mathrm{ab}$ & A3,66a & $\mathrm{B} 3,16 \mathrm{~b}$ & A3,72a & $\mathrm{B} 3,12 \mathrm{~b}$ & $\mathrm{~A} 3,78 \mathrm{a}$ & $\mathrm{B} 3,13 \mathrm{ab}$ & $\mathrm{A} 3,66 \mathrm{a}$ \\
\hline 200 & $\mathrm{~B} 1,23$ & $\mathrm{~A} 2,05 \mathrm{a}$ & $2,98^{\mathrm{a}}$ & $3,22 \mathrm{a}$ & $3,51 \mathrm{a}$ & $3,61 \mathrm{a}$ & $3,67 \mathrm{a}$ & $3,71 \mathrm{a}$ & $3,72 \mathrm{a}$ & $3,67 \mathrm{a}$ & $3,59 \mathrm{a}$ & $3,54 \mathrm{a}$ \\
\hline d.m.s ${ }^{2}$ & \multicolumn{2}{|c|}{0,34} & \multicolumn{2}{|c|}{$0,41^{5,22 \mathrm{~d}}$} & \multicolumn{2}{|c|}{$0,45^{J, 01 a}$} & \multicolumn{2}{|c|}{0,40} & & & & \\
\hline d.m. $s^{3}$ & & & & & & & & & & & & \\
\hline & Cony & ência & Con & ência & Conv & ência & Conv & encia & Con & ncia & Conv & ncia \\
\hline $\begin{array}{l}\text { Dose de } \\
\text { Fósforo } \\
(\mathrm{ppm})\end{array}$ & Sem & Com & Sem & Com & Sem & Com & Sem & Com & Sem & Com & Sem & Com \\
\hline 00 & $1,00 \mathrm{~b}$ & 1,00 & $1,00 \mathrm{c}$ & $1,00 \mathrm{c}$ & $1,00 \mathrm{c}$ & $1,00 \mathrm{~b}$ & $1,00 \mathrm{c}$ & $1,00 \mathrm{~b}$ & $1,00 \mathrm{c}$ & $1,00 \mathrm{~b}$ & $1,15 b$ & $1,05 b$ \\
\hline 50 & $1,09 \mathrm{~b}$ & 1,00 & $\mathrm{~A} 2,76 \mathrm{~b}$ & B1,53b & A $3,74 b$ & $\mathrm{~B} 2,32 \mathrm{a}$ & A3,79b & $\mathrm{B} 2,65 \mathrm{a}$ & A $3,92 b$ & $\mathrm{~B} 2,67 \mathrm{a}$ & A3,96b & B2, $44 \mathrm{a}$ \\
\hline 100 & $\mathrm{~A} 1,89 \mathrm{a}$ & B1,05 & $\mathrm{A} 3,45^{\mathrm{a}}$ & $\mathrm{B} 1,71 \mathrm{~b}$ & $\mathrm{~A} 4,25 \mathrm{a}$ & $\mathrm{B} 2,48 \mathrm{a}$ & $\mathrm{A} 4,30 \mathrm{ab}$ & B2,58a & $\mathrm{A} 4,34 \mathrm{a}$ & B2,57a & $\mathrm{A} 4,36 \mathrm{ab}$ & $\mathrm{B} 2,43 \mathrm{a}$ \\
\hline 200 & $\mathrm{~A} 2,03 \mathrm{a}$ & $\mathrm{B} 1,25$ & $\mathrm{~A} 3,85^{\mathrm{a}}$ & $\mathrm{B} 2,34 \mathrm{a}$ & $\mathrm{A} 4,53 \mathrm{a}$ & B2,59a & $\mathrm{A} 4,59 \mathrm{a}$ & $\mathrm{B} 2,78 \mathrm{a}$ & $\mathrm{A} 4,60 \mathrm{a}$ & $\mathrm{B} 2,78 \mathrm{a}$ & $\mathrm{A} 4,50^{\mathrm{a}}$ & $\mathrm{B} 2,62 \mathrm{a}$ \\
\hline d.m.s ${ }^{2}$ & & & & & & & & & & & & \\
\hline d.m.s ${ }^{3}$ & & & & & & & & & & & & \\
\hline
\end{tabular}


Na Tabela 9, estão apresentados os dados referentes aos efeitos da calagem, da convivência das plantas de B. plantaginea e da dose de fósforo sobre o acúmulo de matéria seca nas diferentes estruturas morfológicas de soja ao final do estudo e, na Tabela 10, os valores obtidos no desdobramento dos graus de liberdade dos tratamentos quando as interações foram significativas.

A calagem influenciou o acúmulo de matéria seca em todas as estruturas morfológicas de soja. Houve incrementos da ordem de 14,8, $20,4,25,2,23,2$ e $22,1 \%$ para raiz, caule, limbo foliar, parte aérea e planta inteira, respectivamente. Os efeitos negativos de plantas daninhas sobre parâmetros de crescimento da soja, ja foram observados por vários pesquisadores, como ( Rossi, 1985; Wulff, 1987 e Velini, 1989).

A convivência com B. plantaginea proporcionou decréscimos no acúmulo de matéria seca nas diferentes estruturas morfológicas de soja e, em média, foram 56,8 , $27,7,28,3,27,5$ e $34,8 \%$ menores em relação a parcela sem convívio, para raiz, caule, limbo foliar, parte aérea e planta inteira, respectivamente.

A adubação fosfatada incrementou o acúmulo de matéria seca nas diferentes partes de soja estudada. A aplicação de $200 \mathrm{ppm}$ de P causou incremento de 156,4, 243,8, 216,3, 233,4 e $208,5 \%$ na biomassa seca da raiz, caule, limbo foliar, parte aérea e planta inteira, respectivamente. Analisando-se os efeitos das doses de fósforo dentro do grupo de tratamentos com e sem calagem, observa-se que tanto no grupo de parcelas com e sem calagem, que já a aplicação de $50 \mathrm{ppm}$ de $\mathrm{P}$ incrementou a biomassa seca das diferentes partes da planta.

A interação entre presença de $B$. plantaginea e as doses de fósforo proporcionaram decréscimo no acúmulo de matéria seca nas raízes, independente da dose utilizada e inclusive na testemunha e, para demais partes da planta este efeito restringiu-se as parcelas que receberam fósforo. Ao analisar- se os efeitos das doses de fósforo dentro dos grupos de tratamentos com e sem convivência de B. plantaginea, nota-se que já a aplicação de 50 ppm de $\mathrm{P}$ ocasionou aumentos no acúmulo de matéria seca de todas as estruturas de soja analisada.

$\mathrm{Na}$ Tabela 11, estão apresentados os dados referentes aos efeitos da calagem, da convivência de plantas de soja e da dose de fósforo sobre o acúmulo de matéria seca nas diferentes estruturas morfológicas de $B$. plantaginea estudadas e, na Tabela 12 , os valores obtidos no desdobramento dos graus de liberdade dos tratamentos quando as interações foram significativas.

A calagem determinou decréscimos no acúmulo de matéria seca de todas as estruturas morfológicas analisadas da planta daninha, com reduções da ordem de 17,5, 26,9, 16,9, 20,6 e $19,2 \%$ para raiz, caule, limbo foliar, parte aérea e planta inteira, respectivamente. A convivência com plantas de soja acarretou reduções na biomassa seca das estrutura morfológicas de $B$. plantaginea da ordem de 25,7, 41,8, 42,1, 43,1 e $36,2 \%$ para raiz, caule, limbo foliar, parte aérea e planta inteira, respectivamente.

Houve efeito da interação calagem e presença de soja sobre o acúmulo de matéria seca de $B$. plantaginea somente para o caule. A calagem determinou efeitos depressivos sobre as plantas que cresceram de forma isolada e a interferência da soja anulou as respostas negativas de $B$. plantaginea à calagem. Independente da realização da calagem às parcelas sem convivência apresentaram acúmulos de matéria seca maiores que nas com convivência.

$O$ uso de fertilizante fosfatado incrementou o acúmulo de matéria seca nas diferentes partes da plantas, independente da dose utilizada, com elevações da ordem de $700,0,259,8,813,6,1.056,9$ e 859,2\% para raiz, caule, limbo foliar, parte aérea e planta inteira, respectivamente. A interação calagem e doses de fósforo só não foi positiva para o acúmulo de matéria seca na raiz. 
Efeito da adubação fosfatada e da calagem nas relações de interferência entre plantas de soja e capim-marmelada

TABELA 9. Valores médios verificados para o acúmulo de matéria seca (g) por vaso em diferentes estruturas morfológicas de plantas de soja, aos 49 dias, obtidos no desdobramento dos graus de liberdade das variáveis principais.

\begin{tabular}{|c|c|c|c|c|c|}
\hline \multirow{2}{*}{ Condição } & \multicolumn{5}{|c|}{ Peso de matéria seca $(\mathrm{g})$} \\
\hline & Raiz & Caule & Limbo foliar & Parte aérea & Planta inteira \\
\hline & \multicolumn{5}{|c|}{ Efeito da Calagem (CA) } \\
\hline $\begin{array}{l}\text { Com } \\
\text { Sem }\end{array}$ & $\begin{array}{l}2,41 a^{1} \\
2,10 b\end{array}$ & $\begin{array}{l}3,36 \mathrm{a} \\
2,79 \mathrm{~b}\end{array}$ & $\begin{array}{l}5,02 \mathrm{a} \\
4,01 \mathrm{~b}\end{array}$ & $\begin{array}{l}8,38 \mathrm{a} \\
6,80 \mathrm{~b}\end{array}$ & $\begin{array}{l}10,78 \mathrm{a} \\
8,83 \mathrm{~b}\end{array}$ \\
\hline Sem & \multicolumn{5}{|c|}{ Efeito da Convivência (CV) } \\
\hline \multirow{3}{*}{\multicolumn{6}{|c|}{ Efeito da dose de Fósforo (DF) }} \\
\hline & & & & & \\
\hline & & & & & \\
\hline 00 & $1,17 \mathrm{c}$ & $1,21 \mathrm{c}$ & $1,90 \mathrm{~d}$ & $3,05 \mathrm{~d}$ & $4,22 \mathrm{c}$ \\
\hline 50 & $2,36 b$ & $3,22 b$ & $4,89 \mathrm{c}$ & $8,02 \mathrm{c}$ & $10,37 \mathrm{~b}$ \\
\hline 100 & $2,51 \mathrm{~b}$ & $3,70 \mathrm{ab}$ & $5,36 \mathrm{~b}$ & $9,12 b$ & $11,62 b$ \\
\hline 200 & $3,00 \mathrm{a}$ & $4,16 \mathrm{a}$ & $6,01 \mathrm{a}$ & $10,17 \mathrm{a}$ & $13,02 \mathrm{a}$ \\
\hline c.v $(\%)$ & 19,05 & 18,04 & 12,04 & 13,08 & 13,05 \\
\hline d.m.s $(\mathrm{CA}$ e CV $)$ & 0,22 & 0,28 & 0,28 & 0,53 & 0,67 \\
\hline d.m.s. (DF) & 0,41 & 0,53 & 0,53 & 0,99 & 1,25 \\
\hline
\end{tabular}

Médias seguidas de mesma letra na coluna não diferem estatisticamente entre si pelo teste de Tukey $(\mathrm{P}>0,05)$

TABELA 10. Valores médios verificados para o acúmulo de matéria seca por vaso em diferentes estruturas morfológicas de plantas de soja, aos 49 dias, obtidos no desdobramento dos graus de liberdade das interações significativas entre calagem e dose de fósforo e, convivência e dose de fósforo.

\begin{tabular}{|c|c|c|c|c|c|c|c|c|c|c|}
\hline \multirow{2}{*}{ Interação } & \multicolumn{10}{|c|}{ Acúmulo da matéria seca $(\mathrm{g})$} \\
\hline & \multicolumn{2}{|c|}{ Raiz } & \multicolumn{2}{|c|}{ Caule } & \multicolumn{2}{|c|}{ Limbo foliar } & \multicolumn{2}{|c|}{ Parte aérea } & \multicolumn{2}{|c|}{ Planta inteira } \\
\hline & \multicolumn{2}{|c|}{ Calagem } & \multicolumn{2}{|c|}{ Calagem } & \multicolumn{2}{|c|}{ Calagem } & \multicolumn{2}{|c|}{ Calagem } & \multicolumn{2}{|c|}{ Calagem } \\
\hline $\begin{array}{l}\text { Dose de } \\
\text { fósforo } \\
(\mathrm{ppm})\end{array}$ & Com & Sem & Com & Sem & Com & Sem & Com & Sem & Com & Sem \\
\hline 00 & $1,21 \mathrm{~b}$ & $1,14 \mathrm{c}$ & $1,19 b$ & $1,24 \mathrm{c}$ & $1,87 \mathrm{~b}$ & $1,94 \mathrm{~d}$ & $3,05 \mathrm{~b}$ & $3,05 \mathrm{~b}$ & $4,26 \mathrm{~b}$ & $4,17 \mathrm{~d}$ \\
\hline 50 & $2,77 \mathrm{a}$ & $1,94 b$ & $\mathrm{~A} 3,84 \mathrm{a}$ & $\mathrm{B} 2,60 \mathrm{~b}$ & A5,76a & $\mathrm{B} 3,84 \mathrm{c}$ & $\mathrm{A} 12,37 \mathrm{a}$ & $\mathrm{B} 8,37 \mathrm{c}$ & $\mathrm{A} 12,37 \mathrm{a}$ & B08,37c \\
\hline 100 & $\mathrm{~A} 2,67 \mathrm{a}$ & $\mathrm{B} 2,34 \mathrm{~b}$ & $\mathrm{~A} 4,17 \mathrm{a}$ & $\mathrm{B} 3,22 \mathrm{~b}$ & A6,10a & $\mathrm{B} 4,61 \mathrm{~b}$ & $\mathrm{~A} 10,27 \mathrm{a}$ & $\mathrm{B} 7,96 \mathrm{~b}$ & A12,94a & B10,30b \\
\hline 200 & $2,97 \mathrm{a}$ & $3,00 \mathrm{a}$ & $4,22 \mathrm{a}$ & 4,09a & A6,36a & B5,66a & $10,59 a$ & $9,75 \mathrm{a}$ & $13,56 \mathrm{a}$ & $12,49 \mathrm{a}$ \\
\hline d.m.s ${ }^{2}$ & \multicolumn{2}{|c|}{0,59} & \multicolumn{2}{|c|}{0,75} & \multicolumn{2}{|c|}{0,75} & \multicolumn{2}{|c|}{1,39} & \multicolumn{2}{|c|}{1,76} \\
\hline d.m.s.s & \multicolumn{2}{|c|}{0,44} & \multicolumn{2}{|c|}{0,57} & \multicolumn{2}{|c|}{0,56} & \multicolumn{2}{|c|}{1,05} & \multicolumn{2}{|c|}{ _. 1,33} \\
\hline $\begin{array}{l}\text { Dose de } \\
\text { fósforo } \\
(\mathrm{ppm})\end{array}$ & Sem & Com & Sem & Com & Sem & Com & Sem & Com & Sem & Com \\
\hline 00 & $\mathrm{~A} 1,55 \mathrm{c}$ & B0,80b & $1,31 \mathrm{c}$ & $1,11 \mathrm{c}$ & $1,89 \mathrm{c}$ & $1,91 \mathrm{~d}$ & $3,07 \mathrm{c}$ & $3,02 \mathrm{c}$ & $4,62 \mathrm{c}$ & $3,81 \mathrm{c}$ \\
\hline 50 & $\mathrm{~A} 3,89 \mathrm{~b}$ & $\mathrm{~B} 1,32 \mathrm{ab}$ & $\mathrm{A} 3,87 \mathrm{~b}$ & $\mathrm{~B} 2,56 \mathrm{~b}$ & A5,97b & $\mathrm{B} 3,62 \mathrm{c}$ & $\mathrm{A} 09,85 \mathrm{~b}$ & $\mathrm{~B} 6,19 \mathrm{~b}$ & $\mathrm{~A} 13,24 \mathrm{~b}$ & $\mathrm{~B} 07,15 \mathrm{~b}$ \\
\hline 100 & $\mathrm{~A} 3,52 \mathrm{a}$ & B1,49a & $\mathrm{A} 4,30 \mathrm{ab}$ & B3,10ab & $\mathrm{A} 6,34 \mathrm{~b}$ & $\mathrm{~B} 4,37 \mathrm{~b}$ & A10,64ab & $\mathrm{B} 7,60 \mathrm{a}$ & $\mathrm{A} 14,15 \mathrm{ab}$ & B09,09ab \\
\hline 200 & $\mathrm{~A} 4,15 \mathrm{a}$ & $\mathrm{B} 1,82 \mathrm{a}$ & $\mathrm{A} 4,79 \mathrm{a}$ & $\mathrm{B} 3,52 \mathrm{a}$ & A6,84a & $\mathrm{B} 5,19 \mathrm{a}$ & $\mathrm{A} 11,62 \mathrm{a}$ & $\mathrm{B} 8,71 \mathrm{a}$ & A15,51a & $\mathrm{B} 10,54 \mathrm{a}$ \\
\hline d.m.s $s^{2}$ & \multicolumn{2}{|c|}{0,59} & \multicolumn{2}{|c|}{0,75} & \multicolumn{2}{|c|}{0,75} & \multicolumn{2}{|c|}{1,39} & \multicolumn{2}{|c|}{1,76} \\
\hline d.m.s $s^{3}$ & \multicolumn{2}{|c|}{0,44} & \multicolumn{2}{|c|}{0,57} & \multicolumn{2}{|c|}{0,56} & 1, & & & 33 \\
\hline
\end{tabular}

${ }_{2}^{1}$ Médias seguidas de mesma letra, minúscula na coluna e maiúscula na linha, não diferem estatisticamente entre si pelo teste de Tukey (P $\left.>0,05\right)$.

2. Comparação na coluna.

${ }^{3}$ - Comparação na linha. 
TABELA 11. Valores médios verificados para o acúmulo de matéria seca por vaso, em diferentes estruturas morfológicas de plantas de Brachiaria plantaginea, aos 49 dias, obtidos no desdobramento dos graus de liberdade das variáveis principais.

\begin{tabular}{|c|c|c|c|c|c|}
\hline \multirow{2}{*}{ Condição } & \multicolumn{5}{|c|}{ Acúmulo de matéria seca $(\mathrm{g})$} \\
\hline & Raiz & Caule & Limbo foliar & Parte aérea & Planta inteira \\
\hline & \multicolumn{5}{|c|}{ Efeito da Calagem (CA) } \\
\hline Com & $3,59 b^{1}$ & $2,55 b$ & $2,71 b$ & $5,25 \mathrm{~b}$ & $8,81 \mathrm{~b}$ \\
\hline \multirow[t]{2}{*}{ Sem } & $4,35 \underline{-} \mathrm{a}_{-}$ & 3, & $3,26 \mathrm{a}$ & $6,61 \mathrm{a}$ & $10,09 \mathrm{a}$ \\
\hline & \multicolumn{5}{|c|}{ Efeito da Convivência (CV) } \\
\hline Sem & $4,55 \mathrm{a}$ & $3,81 \mathrm{a}$ & $3,78 \mathrm{a}$ & $7,56 a$ & $12,05 \mathrm{a}$ \\
\hline \multirow{3}{*}{\multicolumn{6}{|c|}{ Efeito da dose de Fósforo (DF) }} \\
\hline & & & & & \\
\hline & & & & & \\
\hline 00 & $0,62 b$ & $0,24 b$ & $0,44 b$ & $0,68 b$ & $1,30 \mathrm{~b}$ \\
\hline 50 & $4,96 \mathrm{a}$ & $3,59 a$ & $4,02 \mathrm{a}$ & $7,52 \mathrm{a}$ & $12,47 \mathrm{a}$ \\
\hline 100 & $5,34 \mathrm{a}$ & $4,01 \mathrm{a}$ & $3,77 \mathrm{a}$ & $7,84 \mathrm{a}$ & $13,12^{\mathrm{a}}$ \\
\hline 200 & $4,96 \mathrm{a}$ & $4,25 \mathrm{a}$ & $3,70 \mathrm{a}$ & $7,64 a$ & $12,58^{\mathrm{a}}$ \\
\hline c.v $(\%)$ & 19,04 & 23,03 & 25,00 & 23,05 & 20,08 \\
\hline d.m.s (CA e CV) & 0,39 & 0,35 & 0,38 & 0,70 & 1,08 \\
\hline d.m.s. (DF) & 0,72 & 0,66 & 0,71 & 1,31 & 1,88 \\
\hline
\end{tabular}

TABELA 12. Valores médios verificados para o acúmulo de matéria seca por vaso em diferentes estruturas morfológicas de plantas de Brachiaria plantaginea, aos 49 dias, obtidos no desdobramento dos graus de liberdade das interações entre calagem e convivência, calagem e dose de fósforo e, convivência e dose de fósforo.

\begin{tabular}{|c|c|c|c|c|c|c|c|c|c|c|}
\hline \multirow[t]{2}{*}{ Interação } & \multicolumn{10}{|c|}{ Acúmulo da matéria seca $(\mathrm{g})$} \\
\hline & \multicolumn{2}{|c|}{ Raiz } & \multicolumn{2}{|c|}{ Caule } & \multicolumn{2}{|c|}{ Limbo foliar } & \multicolumn{2}{|c|}{ Parte aérea } & \multicolumn{2}{|c|}{ Planta inteira } \\
\hline & \multicolumn{2}{|c|}{ Convivência } & \multicolumn{2}{|c|}{ Convivência } & \multicolumn{2}{|c|}{ Convivência } & \multicolumn{2}{|c|}{ Convivência } & \multicolumn{2}{|c|}{ Convivência } \\
\hline Calagem & Sem & Com & Sem & Com & Sem & Com & Sem & Com & Sem & Com \\
\hline Com & - & - & $\mathrm{A} 3,06 \mathrm{~b}$ & B2,04 & - & - & - & - & - & - \\
\hline Sem & - & - & $\mathrm{A} 4,56 \mathrm{a}$ & B2,42 & - & - & - & - & - & - \\
\hline \multirow[t]{2}{*}{ d.m.s } & \multicolumn{2}{|c|}{ - } & \multicolumn{2}{|c|}{0,50} & \multicolumn{2}{|c|}{ - } & \multicolumn{2}{|c|}{-} & \multicolumn{2}{|c|}{ - } \\
\hline & \multicolumn{2}{|c|}{ Calagem } & \multicolumn{2}{|c|}{ Calagem } & \multicolumn{2}{|c|}{ Calagem } & \multicolumn{2}{|c|}{ Calagem } & \multicolumn{2}{|c|}{ Calagem } \\
\hline $\begin{array}{l}\text { Dose de } \\
\text { fósforo } \\
\text { (ppm) }\end{array}$ & Com & Sem & Com & Sem & Com & Sem & Com & Sem & Com & Sem \\
\hline 00 & - & - & $0,25 b$ & $0,22 b$ & $0,44 \mathrm{~b}$ & $0,45 b$ & $0,69 b$ & $0,67 b$ & $1,25 \mathrm{~b}$ & $1,35 \mathrm{~b}$ \\
\hline 50 & - & - & B2,92a & $\mathrm{A} 4,25 \mathrm{a}$ & B3,46a & $\mathrm{A} 4,59 \mathrm{a}$ & B6,39a & A8,65a & B10,77a & A14,17a \\
\hline 100 & - & - & $2,30 \mathrm{a}$ & $4,30 \mathrm{a}$ & $3,80 \mathrm{a}$ & $3,75 \mathrm{a}$ & $7,47 \mathrm{a}$ & $8,21 \mathrm{a}$ & $12,43 \mathrm{a}$ & $13,80 \mathrm{a}$ \\
\hline 200 & - & - & B3,71a & A5,19a & B3,14a & $\mathrm{A} 4,26 \mathrm{a}$ & B6,44a & A8,92a & B10,76a & A14,40a \\
\hline d.m.s (coluna) & \multicolumn{2}{|c|}{ - } & \multicolumn{2}{|c|}{0,94} & \multicolumn{2}{|c|}{1,00} & \multicolumn{2}{|c|}{1,85} & \multicolumn{2}{|c|}{2,67} \\
\hline \multirow[t]{2}{*}{ d.m.s (linha) } & & & & & & & & & & \\
\hline & \multicolumn{2}{|c|}{ Convivência } & \multicolumn{2}{|c|}{ Convivência } & \multicolumn{2}{|c|}{ Convivência } & Conv & ência & Cony & Vência \\
\hline $\begin{array}{l}\text { Dose de } \\
\text { fósforo } \\
\text { (ppm) }\end{array}$ & Sem & Com & Sem & Com & Sem & Com & Sem & Com & Sem & Com \\
\hline 00 & $0,47 \mathrm{~b}$ & $0,76 \mathrm{~b}$ & $0,17 \mathrm{~b}$ & $0,30 \mathrm{~b}$ & $0,44 \mathrm{~b}$ & $0,45 \mathrm{~b}$ & $0,61 b$ & $0,75 b$ & $1,09 \mathrm{~b}$ & $1,52 b$ \\
\hline 50 & A5,84a & B4,04a & $\mathrm{A} 4,64 \mathrm{a}$ & B2,54a & $\mathrm{A} 5,04 \mathrm{a}$ & $\mathrm{B} 3,10 \mathrm{a}$ & A9,49a & B5,55a & A15,32a & B9,62a \\
\hline 100 & $\mathrm{~A} 6,47 \mathrm{a}$ & $\mathrm{B} 4,20 \mathrm{a}$ & A5,40a & $\mathrm{B} 2,61 \mathrm{a}$ & $\mathrm{A} 4,94 \mathrm{a}$ & $\mathrm{B} 2,61 \mathrm{a}$ & A10,52a & $\mathrm{B} 5,16 \mathrm{a}$ & A16,87a & B9,36a \\
\hline 200 & A5,49a & $\mathrm{B} 4,50 \mathrm{a}$ & $\mathrm{A} 5,02 \mathrm{a}$ & B3,46a & $\mathrm{A} 4,70 \mathrm{a}$ & B2,70a & A9,60a & B5,76a & A14,90a & B10,26a \\
\hline d.m.s (coluna) & & & & & & & & & & \\
\hline d.m.s (linha) & & 77 & & & & 75 & & & & \\
\hline
\end{tabular}


A calagem proporcionou decréscimos no acúmulo de matéria seca nas diferentes estruturas morfológicas de $B$. plantaginea apenas no grupo de parcelas que receberam 50 e $200 \mathrm{ppm}$ de P. Ao comparar-se os efeitos das doses de fósforo dentro dos grupos de tratamentos com e sem calagem, nota-se incrementos no acúmulo de matéria já com aplicação de 50 ppm de P.

A interação entre presença de soja e as doses de fósforo proporcionou decréscimos no acúmulo de matéria seca nas estruturas morfológicas de plantas de B. plantaginea quando da presença da soja, independente da dose de fósforo estudada. Ao comparar-se os efeitos das doses de fósforo dentro dos grupos de tratamentos com e sem calagem, não se verificou respostas diferenciadas entre as doses testadas $(50,100 \mathrm{e}$ 200 ppm).

Os efeitos da interferência da soja sobre $B$. plantaginea apenas foram significativos nas parcelas que receberam a adubação fosfatada. Tal comportamento, foi indício importante de que os teores de $\mathbf{P}$ no solo constituem-se em fatores ecológicos limitantes que atuam em maior grau do que a própria presença da planta de soja. A soja passou a interferir significativamente quando houve fornecimento do nutriente. Por outro lado, deve-se destacar a forte interferência das plantas de soja na redução das respostas das plantas de $B$. plantaginea à adubação fosfatada.

Outro indício de que o nível de $\mathrm{P}$ era o fator preponderante no crescimento da gramínea está na resposta negativa à calagem observada nas parcelas que receberam doses intermediárias de adubação fosfatada quando o fornecimento de carbonato de cálcio passou a limitar $\mathrm{p}$ sei crescimento.

Na Tabela 13, estão apresentados os dados referentes aos efeitos da calagem, da convivência e da dose de fósforo sobre a área foliar (AF), bem como os valores médios obtidos nos desdobramentos dos graus de liberdades dos tratamentos quando as interações foram significativas.

A calagem incrementou apenas a $\mathrm{AF}$ da soja, com um aumento médio de $32,4 \%$. A convivência influenciou ambas as espécies. A interferência imposta pela $B$. plantaginea acarretou uma redução de $26,3 \%$ na AF da soja e, a interferência imposta pela soja determinou decréscimos da ordem de 30,7\% em sua AF.

Reduções na AF da soja também foram verificadas por (Cordes \& Bauman, 1984; Wyse et al., 1986 e Velini, 1989). Para Velini (1989) a AF consiste-se no parâmetro mais sensível para evidenciar respostas de plantas ao processo de interferência e, tal evidência tem sido verificado em outros trabalhos conduzidos com diferentes culturas, como eucaliptus (Marchi, 1989) e cevada (Souza, 1992).

A interação entre calagem e convivência não influenciou a $\mathrm{AF}$ da soja, porém para $B$. plantaginea houve respostas. A calagem proporcionou incrementos na $\mathrm{AF}$ da planta daninha do grupo de parcelas com convivência com soja e, independente da realização da calagem ou não, a convivência com a soja determinou decréscimos na AF da planta daninha no grupo de parcelas com convivência.

A adubação fosfatada incrementou a $\mathrm{AF}$ da soja em média 198,6\% com à aplicação de 200 ppm de $\mathrm{P}$ e $298 \%$ em relação à $\mathrm{AF}$ de $B$. plantaginea. A interação entre calagem e doses de fósforo foi significativa. A calagem incrementou a $\mathrm{AF}$ da soja nas parcelas que receberam a aplicação de fósforo. Observa-se, ainda, que independente da realização da calagem, houve incrementos com a realização da adubação.

A interação entre convivência e doses de fósforo foi significativa para ambas as espécies. Nota-se, tanto para a soja como para planta daninha, que a convivência promoveu decréscimos na $\mathrm{AF}$ no grupo de parcelas que receberam a aplicação de $\mathrm{P}$ e quando se comparam os efeitos da adubação com dentro do de tratamentos com sem convivência, verifica-se que à adubação fosfatada proporcionou ganhos em ambos os grupos de tratamentos.

$\mathrm{Na}$ Tabela 14 podem ser observados os efeitos da calagem, da convivência a dose de fósforo sobre os teores de clorofila $\mathbf{a}$ e $\mathbf{b}$ e, na Tabela 15,os valores obtidos nos desdobramentos dos graus de liberdade dos tratamentos quando as interações foram significativas. 
TABELA 13. Valores médios verificados para área foliar (A.F.) de soja e Brachiaria plantaginea, aos 49 dias, obtidos no desdobramento dos graus de liberdade das variáveis principais e das interações entre calagem e convivência, dose de fósforo e calagem e convivência e dose de fósforo.

\begin{tabular}{|c|c|c|c|c|c|c|c|c|}
\hline \multirow{2}{*}{ Condição } & \multicolumn{2}{|c|}{ A. F. $\left(\mathrm{dm}^{2}\right)$} & \multicolumn{4}{|c|}{ A. F. $\left(\mathrm{dm}^{2}\right)$} & \multirow{2}{*}{\multicolumn{2}{|c|}{$\begin{array}{c}\text { A. F. }\left(\mathrm{dm}^{2}\right) \\
\text { Soja }\end{array}$}} \\
\hline & BRAPL & Soja & & & & & & \\
\hline \multirow{2}{*}{\multicolumn{3}{|c|}{ Efeito da Calagem }} & & & & & \multicolumn{2}{|c|}{ Calagem } \\
\hline & & & & & & Dose de Fósforo & Com & Sem \\
\hline Com & 10,43 & $16,30 \mathrm{a}^{1}$ & & & & $(\mathrm{ppm})$ & & \\
\hline \multirow{3}{*}{ Sem } & 10,11 & $12,32 \mathrm{~b}$ & & & & 00 & $6,29 b$ & $6,45 c$ \\
\hline & Efeito d & vência & & Convi & vência & 50 & A19,19a & B $11,90 \mathrm{~b}$ \\
\hline & & & Calagem & Sem & Com & 100 & A19,29a & B $13,28 b$ \\
\hline Sem & $12,13 \mathrm{a}$ & $16,48 \mathrm{a}$ & Com & A 11,85 & B9,01a & 200 & A $20,41 \mathrm{a}$ & $\mathrm{B} 17,63 \mathrm{a}$ \\
\hline \multirow{2}{*}{ Com } & $8,41 \mathrm{~b}$ & $12,14 \mathrm{~b}$ & Sem & A12,41 & $\mathrm{B} 7,81 \mathrm{~b}$ & d.m.s. (coluna) & \multicolumn{2}{|c|}{2,17} \\
\hline & \multicolumn{2}{|c|}{ Efeito da dose de Fósforo } & d.m.s. & 1 & & d.m.s. (linha) & \multicolumn{2}{|c|}{1,64} \\
\hline \multicolumn{3}{|l|}{ (ppm) } & \multicolumn{4}{|c|}{ Convivência } & \multicolumn{2}{|c|}{ Convivência } \\
\hline 00 & $2,89 \mathrm{c}$ & $6,37 \mathrm{c}$ & Dose de Fósforo & Sem & Com & Dose de Fósforo & Sem & Com \\
\hline 50 & $14,37 \mathrm{a}$ & $15,55 b$ & $(\mathrm{ppm})$ & & & $(\mathrm{ppm})$ & & \\
\hline 100 & $12,29 b$ & $16,28 b$ & 00 & $2,080 \mathrm{~b}$ & $2,99 \mathrm{c}$ & 00 & $6,44 b$ & $6,30 \mathrm{c}$ \\
\hline 200 & $11,52 \mathrm{~b}$. & $19,02 \mathrm{a}$ & 50 & A $15,89 a$ & B12,85a & 50 & A19,23a & B $11,86 b$ \\
\hline c.V.\% & 15,07 & 11,04 & 100 & A $15,57 \mathrm{a}$ & B $9,02 b$ & 100 & A19,19a & B $13,38 b$ \\
\hline d.m.s (CA) & 0,81 & 0,82 & 200 & A14,27ab & B $8,77 \mathrm{~b}$ & 200 & A $21,04^{\mathrm{a}}$ & B17,01a \\
\hline d.m.s (CV) & 0,81 & 0,82 & d.m.s (coluna) & & & d.m.s. (coluna) & & \\
\hline d.m.s (DF) & 1,51 & 1,53 & d.m.s. (linha) & 1 , & & d.m.s. (linha) & & \\
\hline
\end{tabular}

${ }^{1}$ Médias seguidas de mesma letra, minúscula na coluna e maiúscula na linha, não diferem estatisticamente entre si pelo teste de Tukey (P>0,05).

TABELA 14. Valores médios verificados para o conteúdo de clorofila a e b nas folhas de soja e Brachiaria plantaginea, aos 49 dias obtidos no desdobramento dos graus de liberdade das variáveis principais.

\begin{tabular}{|c|c|c|c|c|}
\hline \multirow[b]{2}{*}{ Condição } & \multicolumn{4}{|c|}{ Teor de clorofila (mg/g de matéria verde) } \\
\hline & $\begin{array}{c}\mathbf{a} \\
\text { Soia }\end{array}$ & $\begin{array}{c}\mathbf{a} \\
\mathrm{BRAPI}\end{array}$ & b & $\begin{array}{c}\mathbf{b} \\
\mathrm{BR} A \mathrm{PI}\end{array}$ \\
\hline & \multicolumn{4}{|c|}{ Efeito da calagem $(\mathrm{CA})$} \\
\hline Com & $0,69 a^{1}$ & $0,45^{\mathrm{a}}$ & $0,54 \mathrm{a}$ & $0,37 \mathrm{a}$ \\
\hline \multirow[t]{2}{*}{ Sem } & $0,55 b$ & $0,41 b$ & $0,42 b$ & $0,30 \mathrm{~b}$ \\
\hline & \multicolumn{4}{|c|}{ Efeito da convivência (CV) } \\
\hline Sem & $0,66 \mathrm{a}$ & $0,44^{\mathrm{a}}$ & $0,53 \mathrm{a}$ & $0,36 \mathrm{a}$ \\
\hline \multirow[t]{2}{*}{ Com } & $0,59 \mathrm{~b}$ & $0,41 \mathrm{~b}$ & $0,42 b$ & $0,31 \mathrm{~b}$ \\
\hline & \multicolumn{4}{|c|}{ Efeito da dose de Fósforo (DF) } \\
\hline$(\mathrm{ppm})$ & & & & \\
\hline 00 & $0,70 \mathrm{a}$ & $0,62 \mathrm{a}$ & $0,55 \mathrm{a}$ & $0,48 \mathrm{a}$ \\
\hline 50 & $0,64 b$ & $0,38 b$ & $0,51 b$ & $0,29 b b$ \\
\hline 100 & $0,60 \mathrm{c}$ & $0,34 \mathrm{c}$ & $0,44 \mathrm{c}$ & $0,27 \mathrm{c}$ \\
\hline 200 & $0,53 \mathrm{~d}$ & $0,39 \mathrm{~b}$ & $0,40 \mathrm{~d}$ & $0,30 \mathrm{~b}$ \\
\hline c.v. $(\%)$ & 6,07 & 9,00 & 6,07 & 19,01 \\
\hline d.m.s. (CA e CV) & 0,02 & 0,02 & 0,02 & 0,02 \\
\hline d.m.s. (DF) & 0,04 & 0,04 & 0,03 & 0,03 \\
\hline
\end{tabular}

${ }^{1}$ Médias seguidas de mesma letra na coluna não diferem estatisticamente entre si pelo teste de Tukey $(\mathrm{P}>0,05)$.

BRAPL - Brachiaria plantaginea 
TABELA 15. Valores médios verificados para o conteúdo de clorofila a e b nas folhas de soja e Brachiaria plantaginea aos 49 dias obtidos no desdobramento dos graus de liberdade das interações entre calagem e convivência, calagem e dose de fósforo e, convivência e dose de fósforo.

\begin{tabular}{|c|c|c|c|c|c|c|c|c|}
\hline \multirow[b]{2}{*}{ Interação } & \multicolumn{8}{|c|}{ Teor de clorofila (mg/g de matéria verde) } \\
\hline & \multicolumn{2}{|c|}{$\begin{array}{c}\text { a } \\
\text { Soja }\end{array}$} & \multicolumn{2}{|c|}{$\begin{array}{c}\mathbf{a} \\
\text { BRAPL }\end{array}$} & \multicolumn{2}{|c|}{$\begin{array}{c}\text { b } \\
\text { soja }\end{array}$} & \multicolumn{2}{|c|}{$\begin{array}{c}\mathbf{b} \\
\text { BRAPL }\end{array}$} \\
\hline & \multicolumn{2}{|c|}{ Convivência } & \multicolumn{2}{|c|}{ Convivência } & \multicolumn{2}{|c|}{ Convivência } & \multicolumn{2}{|c|}{ Convivência } \\
\hline Calagem & Sem & Com & Sem & Com & Sem & Com & Sem & Com \\
\hline Com & - & - & $\mathrm{A} 0,48 \mathrm{a}$ & B0,43 & - & - & A0,41a & B0,33a \\
\hline Sem & - & - & $0,41 b$ & 0,41 & - & - & $0,31 \mathrm{~b}$ & $0,29 b$ \\
\hline \multirow[t]{2}{*}{ d.m.s. } & \multicolumn{2}{|c|}{-1} & \multicolumn{2}{|c|}{0,03} & & & \multicolumn{2}{|c|}{0,02} \\
\hline & \multicolumn{2}{|c|}{ Calagem } & \multicolumn{2}{|c|}{ Calagem } & \multicolumn{2}{|c|}{ Calagem } & \multicolumn{2}{|c|}{ Calagem } \\
\hline $\begin{array}{l}\text { Dose de Fósforo } \\
(\mathrm{ppm})\end{array}$ & Sem & Com & Sem & Com & Sem & Com & Sem & Com \\
\hline 00 & $0,72 \mathrm{a}$ & $0,62 \mathrm{a}$ & - & - & $0,55 \mathrm{a}$ & $0,55 \mathrm{a}$ & A0,56a & B0,39a \\
\hline 50 & A0,68ab & B0,61b & - & - & A0,55a & B $0,47 \mathrm{~b}$ & $A 0,32 b$ & B0,27a \\
\hline 100 & A0,69ab & $\mathrm{B} 0,51 \mathrm{c}$ & - & - & A0,54ab & $\mathrm{B} 0,34 \mathrm{c}$ & $0,27 \mathrm{c}$ & $0,26 \mathrm{~b}$ \\
\hline 200 & $\mathrm{~A} 0,65 \mathrm{~b}$ & B0,41d & - & - & $\mathrm{A} 0,50 \mathrm{~b}$ & B0,31c & A0,33b & B0,28b \\
\hline d.m.s. (coluna) & \multicolumn{2}{|c|}{0,05} & \multicolumn{2}{|c|}{-} & \multicolumn{2}{|c|}{0,04} & \multicolumn{2}{|c|}{0,04} \\
\hline d.m.s.(linha) & \multicolumn{2}{|c|}{0,04} & \multicolumn{2}{|c|}{$-\ldots \ldots$} & \multicolumn{2}{|c|}{0,03} & \multicolumn{2}{|c|}{0,03} \\
\hline & \multicolumn{2}{|c|}{ Convivência } & \multicolumn{2}{|c|}{ Convivência } & \multicolumn{2}{|c|}{ Convivência } & \multicolumn{2}{|c|}{ Convivência } \\
\hline $\begin{array}{l}\text { Dose de Fósforo } \\
(\mathrm{ppm})\end{array}$ & Sem & Com & Sem & Com & Sem & Com & Sem & Com \\
\hline 00 & A0,75a & B0,66a & - & - & $\mathrm{A} 0,58 \mathrm{a}$ & B0,52a & - & - \\
\hline 50 & A0,70a & $\mathrm{B} 0,59 \mathrm{~b}$ & - & - & A0,60a & $\mathrm{B} 0,43 \mathrm{~b}$ & - & - \\
\hline 100 & $0,61 b$ & $0,59 b$ & - & - & $\mathrm{A} 0,49 \mathrm{~b}$ & B0,39bc & - & - \\
\hline 200 & $\mathrm{~A} 0,56 \mathrm{~b}$ & B 0,50 & - & - & $\mathrm{A} 0,45 \mathrm{~b}$ & B0,36c & - & - \\
\hline d.m.s. (coluna) & \multicolumn{2}{|c|}{0,05} & \multirow{2}{*}{\multicolumn{2}{|c|}{-}} & \multicolumn{2}{|c|}{0,04} & & \\
\hline d.m.s.(linha) & & & & & 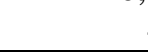 & & & \\
\hline
\end{tabular}

A calagem incrementou os teores de clorofila a e b, tanto das folhas de soja como de $B$. plantaginea e foram em média $25,5 \%$ e $28,6 \%$ maiores para os teores de clorofila a e b da soja e $9,8 \%$ e $23,3 \%$ para os teores de clorofila a e b da planta daninha.

A interferência imposta pela planta daninha proporcionou reduções de 10,6 e 20,5\% no acúmulo de clorofila $\mathbf{a}$ e $\mathbf{b}$ nas folhas de soja, respectivamente, Já, a interferência da soja, acarretou reduções de 6,8 e 13,9\% nos acúmulos de clorofila a e b nas folhas da planta daninha, respectivamente.
A interação entre calagem e convivência influenciou apenas os teores de clorofila a e b da planta daninha. Para clorofila a, nota-se que a calagem incrementou os seus teores no grupo de parcelas sem convivência. A interferência da soja acumulou as respostas da gramínea à calagem, mas para a clorofila b este efeito não foi observado. Em ambos teores de clorofila, a calagem proporcionou valores maiores no grupo de parcelas mantidas livres da interferência da soja e, a não realização da calagem anulou estas respostas. 
$\mathrm{Na}$ interação entre calagem e doses de fósforo, a calagem incrementou os teores de clorofila a e b na soja e b na planta daninha, no grupo de parcelas que recebeu a aplicação de fósforo, exceto para a dose de $100 \mathrm{ppm}$ de $\mathrm{P}$ para o teor de clorofila $\mathbf{b}$ de $B$. plantaginea. Verifica-se, ainda, que os teores de clorofila $\mathbf{a}$ e b apresentaram decréscimos devido a aplicação de pelo menos uma dose de fósforo, independente da realização da calagem ou não.

A interação entre convivência e doses de fósforo influenciou apenas o teor de clorofila $\mathbf{a}$ e $\mathbf{b}$ da soja, sendo que a convivência com a planta daninha, proporcionou reduções nos valores no grupo de parcelas que receberam ou não à aplicação de fósforo. Ao comparar-se os valores dentro de cada grupo nota-se que independente da convivência, os teores de clorofila a e b foram reduzidos com a aplicação do adubo fosfatado.

\section{LITERATURA CITADA}

ARNON, D.I. Copper enzimes in isolated chloroplasts. Polyphenoloxidase in Beta vulgaris. Plant Physiol., v.24, p.1-15, 1949.

BUCHANAN, G.A, MOVELAND, C.S., HARRIS, M.C. Responses of weeds to soil pH. Weed Sci., v.23, n.6, p.473-77, 1975.

CAMARGO, A.P., SOARES, S.T., MAGALHÃES, O.B. Efeito da calagem nas produçõesde cinco cultivares de milho, seguidos de algodão e soja. Pesq. Agropec. Bras., v.17, n.7, p.1.007-12, 1982.

CORDES, R.C., BAUMAN, T.T. Field competition betwen ivyleaf morninglory (Ipomoea hederacea) and soybeans (Glycine max). Weed Sci., v.32, n.3, p.36470, 1984.

GILBERT, B.E., PEMBER, F.R. Tolerance of certain weeds and grasses to toxic aluminium. Soil Sci., v.39, n.5, p.425-9, 1975.
KISMMANN, K.G. Plantas nocivas e infestantes. Ed.Basf, tomo1.1.991.p.31721.

LEGUIZAMON, E.S., BODRERD, M.L., MACOR, L. Interference and control of purple nutsedge (Cyperus rotundus L.) in soybean. Malezas, v.11, n.4, p.209-216, 1983.

MARCHI, S.R. Estudos básicos das relações de interferência entre plantas daninhas $e$ plantas de eucalipto. Jaboticabal/SP: FCAV/UNESP, 1989. 57p. Trabalho (Graduação em Agronomia).

MARTINS, D. Interferência de capim-marmelada na cultura da soja. Planta Daninha, v. 12, n.2, p.93-99, 1994.

MICHILINI, L.A. Nutrição mineral de plantas daninhas. Absorção e distribuição de nutrientes em Bidens pilosa e Brachiaria plantaginea e efeitoss do $\mathrm{pH}$ do solo nos teores de nutrientes em Bidens pilosa e Echinochloa colonum. Jaboticabal/SP: FCAV/UNESP, $1983 . \quad 83 p$. Trabalho (Graduação em Agronomia).

NAKAYAMA, L.H.I., BARBO, C.V.S., FABRILIO, A.C. Aplicação de calcário em Latossolo Roxo sob cultura de soja. Rev. Bras. Cien. Sol., v.8, n.2, p.309-12, 1984.

PITELLI, R.A. Interferência de plantas daninhas em culturas agrícolas. Inf. Agropec., v.11, n.129, p.16-25, 1985.

RAIJ, B.Van., GUAGGIO, J.A. Método de análise de solo para fins de fertilidade. Campinas: Instituto Agronômico, 1983. 31p. (Boletim Técnico, 81).

RAIJ, B.Van., GUAGGIO, J.A., MASCARENHAS, H.A.A. Efeito de níveis de calagem na produção da soja em solo de 
cerrado. Rev. Bras. Cien. Sol., v.1, n.1, p.28-31, 1977.

ROSSI, C.A. Efeitos de períodos de controle e de convivência de plantas daninhas na cultura da soja (Glycine max). Jaboticabal/SP: FCAV/UNESP, 1985. 40p. Trabalho (Graduação em Agronomia).

SAMARRÃO, S.S., BARROS, F.T., ANDRADE, S.T. Influência da calagem e micronutrientes na nodulação da soja pôr Rhizobium japonicum, em solos ácidos. Pesq. Agropecu. Bras., v.21, n.3, p.237-44, 1986.

SOUZA, J.R.P. Efeitos de periodos de controle e de convivência das plantas daninhas na cultura da cevada (Hordeum vulagre $\mathrm{L}$.). Botucatu/SP: FCA/UNESP, 1992. 112p. Trabalho (Dissertação de Mestrado).
VELINI, E.D. Avaliação dos efeitos de comunidades infestantes naturais controladas por diferentes períodos, sobre o crescimento e produtividade da cultura da soja. Jaboticabal/SP: FCAV/UNESP, 1985. 40p. Trabalho (Dissertação de Mestrado).

WULFF, L.T. Growth resonses of soybean (Glycine max) and sorghum (Sorghum bicolor) to na increase in density of Amaranthus dubius L. plants at two temperatures. Weed Res., v.27, n.2, p.7985, 1987.

WYSE, D.L., YONG, F.L., JONES, R.J. Influence of jerusalem artichoke (Helianthus tuberosus) density and duration of interference on soybean growth and yield. Weed Sci., v.34, n.2, p.243-47, 1986. 